Ecología

\title{
Distribución, ciclo biológico y tabla de vida de Eumaeus toxea (Lepidoptera: Lycaenidae) en la provincia fisiográfica Costa de Oaxaca, México
}

\author{
Distribution, biological cycle and life table of Eumaeus toxea (Lepidoptera: Lycaenidae) in the \\ physiographic province Coast of Oaxaca, Mexico
}

\author{
Noe Ruiz-García ${ }^{\mathrm{a}, *}$, Brenda Yesenia Méndez-Pérez ${ }^{\mathrm{b}}$, Mario Valerio Velasco-García ${ }^{\mathrm{a}}$, \\ Guillermo Sánchez-de la Vega ${ }^{a}$ y Juana Laura Rivera-Nava ${ }^{c}$ \\ a Instituto de Ecología, Universidad del Mar, km 3.5 Carretera Puerto Escondido-Oaxaca vía Sola de Vega, 71980 San Pedro Mixtepec, Oaxaca, México \\ ${ }^{\mathrm{b}}$ Ingeniería Forestal, Universidad del Mar, km 3.5 Carretera Puerto Escondido-Oaxaca vía Sola de Vega, 71980 San Pedro Mixtepec, Oaxaca, México \\ ${ }^{c}$ Instituto de Genética, Universidad del Mar, km 3.5 Carretera Puerto Escondido-Oaxaca vía Sola de Vega, 71980 San Pedro Mixtepec, Oaxaca, México
}

Recibido el 13 de agosto de 2014; aceptado el 28 de mayo de 2015

Disponible en Internet el 21 de octubre de 2015

\begin{abstract}
Resumen
La mariposa Eumaeus toxea es un herbívoro que se alimenta de Zamia paucijuga, una cícada considerada amenazada y vulnerable, con una tendencia a la disminución y desaparición de sus poblaciones. Los objetivos del presente trabajo fueron determinar la distribución geográfica de la mariposa, la duración de su ciclo biológico, su supervivencia y reproducción en su hospedante natural en condiciones de campo, en la provincia fisiográfica Costa de Oaxaca. El trabajo se realizó durante el periodo de junio de 2010 a mayo de 2011. La distribución de la mariposa fue coincidente con la distribución de su hospedante. Empleando la técnica de tablas de vida con decrementos simples y agrupados por estadios, se requirió $47 \pm 0.5$ días a la urgencia del adulto, un tiempo generacional de 51 días, una supervivencia de 0.027 hasta la urgencia del adulto con una mortalidad del $28.4 \%$ para el estadio de huevo y del $68.8 \%$ para el estadio de larva; con una tasa de reproducción neta de 0.014 . Por su especificidad con el hospedante, baja densidad poblacional y tasa neta de reproducción, la conservación E. toxea depende de la preservación de su hospedante y del dosel arbóreo donde sobreviven sus poblaciones.

Derechos Reservados ( 2014 Universidad Nacional Autónoma de México, Instituto de Biología. Este es un artículo de acceso abierto distribuido bajo los términos de la Licencia Creative Commons CC BY-NC-ND 4.0.
\end{abstract}

Palabras clave: Cícadas; Endemismo; Zamia paucijuga; Supervivencia; Reproducción

\begin{abstract} depends on the preservation of its host and the canopy where their populations survive. Creative Commons CC License BY-NC-ND 4.0.

Keywords: Cycads; Endemism; Zamia paucijuga; Survival; Reproduction

\footnotetext{
* Autor para correspondencia.

Correo electrónico: nruizg @ zicatela.umar.mx (N. Ruiz-García).

La revisión por pares es responsabilidad de la Universidad Nacional Autónoma de México.
}

The butterfly Eumaeus toxea is an herbivore that feeds on Zamia paucijuga, an endangered and vulnerable cycad with their populations trending to decline and disappear. The objectives of this study were to determine the geographic distribution of the butterfly and to determine the duration of their life cycle, as well as its survival and reproduction rate when reared with its natural host under field conditions in the physiographic province of Oaxaca Coast. The work was conducted during the period of June 2010 to May 2011. Butterfly distribution coincided with the distribution of their host. Using the technique of single decrement and abridged life table by stages, $47 \pm 0.5$ days were required for adult emergence, a mean generation time of 51 days, a survival rate of 0.027 to adult emergence with a mortality of $28.4 \%$ for the egg stage and $68.8 \%$ for the larval stage, and a net reproductive rate of 0.014 . Due to the host specificity, low population density and net reproduction rate, the conservation of $E$. toxea

All Rights Reserved (C 2014 Universidad Nacional Autónoma de México, Instituto de Biología. This is an open access item distributed under the 


\section{Introducción}

La mariposa pequeña de las cícadas, Eumaeus toxea Godart (sin. minyas Hübner) (Lepidoptera: Lycaenidae), es una especie herbívora especialista asociada a las cícadas en el continente americano (Schneider, Wink, Sporer y Lounibos, 2002). Se ha reportado que las larvas de esta mariposa se alimentan de hojas y estróbilos femeninos de varias especies del género Zamia (Cycadales: Zamiacea): Zamia furfuracea L. F. ex Aiton en la vertiente del golfo de México (Castillo-Guevara y Rico-Gray, 2002), Zamia paucijuga Wieland en la vertiente del Pacífico mexicano (Rivera-Nava et al., 2011) y Zamia encephalartoides D. W. Stev., en Colombia (González, 2004). La cícada Z. paucijuga se reporta desde Nayarit hasta Oaxaca (Nicolalde-Morejón, Vovides y Stevenson, 2009). En Oaxaca se ha encontrado en la provincia biogeográfica Planicie Costera (Salas-Morales, Saynes-Vásquez y Schibli, 2003). Específicamente, en las regiones políticas Costa y Sierra Sur se ha reportado en 24 localidades de 9 municipios, en altitudes de 60 a $935 \mathrm{~m}$ snm, asociada a bosques de pino, de encino y tropical subcaducifolio. Sus poblaciones están fragmentadas y las condiciones en donde habitan varían desde sitios conservados con alta densidad de vegetación arbórea hasta sitios sin dosel arbóreo, donde se desarrolla la agricultura (rozatumba-quema), la ganadería extensiva, la cafeticultura y las plantaciones forestales (Velasco-García, Sánchez-de la Vega y Molina-García, 2011). Esta cícada y sus especies asociadas están sujetas a protección especial por la Norma Oficial Mexicana Nom-059-Semarnat-2010 (Semarnat, 2010). En contraste con la biología de relación simbiótica cícadas-polinizadores que ha sido más estudiada (Marler, 2010; Stevenson, Norstog y Fawcett, 1998; Terry et al., 2005), se sabe poco sobre la interacción antagonista entre $Z$. paucijuga y E. toxea, en particular sobre la biología del herbívoro.

El objetivo del presente trabajo fue determinar la distribución geográfica de E. toxea, sus hábitos de alimentación y dar información básica sobre los parámetros reproductivos de esta mariposa, en la provincia fisiográfica Costa de Oaxaca. En particular, se describe su ciclo de vida, estimando la duración de su ciclo biológico, su supervivencia y la reproducción neta en su hábitat natural.

\section{Materiales y métodos}

Durante el periodo de junio de 2010 a mayo de 2011 se realizaron recorridos de prospección en los distritos de Jamiltepec, Juquila y Pochutla del estado de Oaxaca, en aquellas comunidades donde sus pobladores reportaron la presencia de $Z$. paucijuga. Con apoyo de un geoposicionador se registraron las coordenadas geográficas de un sitio de muestreo de $20 \times 20 \mathrm{~m}$ en cada localidad. En cada cuadrante de muestreo se registró la siguiente información: presencia de la mariposa, número de plantas de Z paucijuga con signos de herbivoría y porcentaje de herbivoría en cada planta, así como altitud, el tipo de vegetación natural (Rodarte-García, 1997; Torres-Colín, 2004) y grado de conservación de la vegetación natural del sitio de acuerdo con el siguiente criterio: conservado $(\geq 60 \%$ de cobertura de vegetación arbórea), alterado ( $\leq 60 \%$ de cobertura de vegetación arbórea con actividades de cafeticultura y/o ganadería extensiva) y deforestado ( con actividades de agricultura y/o potreros).

La localidad de $Z$. paucijuga donde se realizó el estudio de su ciclo de vida se localiza en el kilómetro 15 de la carretera Puerto Escondido-Oaxaca ( $\left.16^{\circ} 1^{\prime} 47.1^{\prime \prime} \mathrm{N}, 97^{\circ} 3^{\prime} 59.9^{\prime \prime} \mathrm{O}\right)$, tiene una extensión aproximada de 4.9 ha, elevación de 650 a $850 \mathrm{~m}$ snm y la vegetación corresponde a una zona de transición de bosque de encino con selva baja subcaducifolia. El clima de la localidad es cálido subhúmedo (Aw2[w]), temperatura media anual de $26^{\circ} \mathrm{C}$ y precipitación promedio anual de $1,500 \mathrm{~mm}$ (Inegi, Conabio e INE, 2008).

Mediante revisiones diarias en todas las plantas de Z. paucijuga de la localidad se ubicaron las oviposiciones de E. toxea. Cada puesta fue considerada una cohorte a la cual se le dio seguimiento diario registrando los días que duró cada estadio, número de individuos que pasaron al siguiente estadio y hábitos de alimentación. En las larvas también se registraron los mismos datos para cada subestadio larval. Todas las larvas que llegaron al estadio de pupa de cada cohorte fueron encerradas en jaulas de malla mosquitera de $1.25 \times 1.25 \times 1.25 \mathrm{~m}$ para determinar posteriormente la proporción de sexos de los adultos que emergieron y el número de huevos ovipositados por hembra. Las mariposas fueron alimentadas con pulpa de mango y plátano fermentados.

Empleando la metodología de tablas de vida agrupados por estadios con decrementos simples (Carey, 1993), se estimó la supervivencia por estadio (huevo, larva, pupa, adulto) en el caso de la larva, además, se estimó para cada uno de los 4 subestadios larvales y la prepupa, así como la tasa neta de reproducción y el tiempo medio de cada generación. La tabla de vida se determinó a partir de 74 huevos correspondientes a 12 cohortes, en el periodo de julio a octubre de 2010. Las larvas se alimentaron de las hojas de la planta de Z. paucijuga, donde estuvo la puesta de la cual emergieron. En la temporada seca, que fue de noviembre de 2010 a mayo de 2011, solamente se contaron las oviposiciones y el número de huevos por oviposición.

\section{Resultados}

En todas las localidades donde se encontró el hospedante Z. paucijuga también se encontró el herbívoro E. toxea, por lo que la distribución geográfica de E. toxea fue coincidente con la distribución de su hospedante, en altitudes de 90 hasta $800 \mathrm{~m}$ snm y asociado a 3 tipos de vegetación natural presentes en la región: bosque de encino, selva mediana subcaducifolia y selva baja caducifolia (fig. 1; tabla 1). El porcentaje promedio de herbivoría en cada localidad de Z. paucijuga estuvo directamente relacionado con la densidad poblacional de la planta hospedante y del grado de alteración del dosel arbóreo de cada localidad (fig. 2). Fue en las localidades conservadas donde se observó mayor tasa de herbivoría (de hasta el $48.1 \%$ en la localidad 4, ubicada en bosque de encino dedicado al pastoreo extensivo de ganado caprino), mientras que en los sitios alterados (del 20.5 en el sitio 14) y deforestados (del $26.5 \%$ en el sitio 12) fueron menores, ambos ubicados en remanentes de bosque de encino dedicados a la ganadería bovina intensiva. 


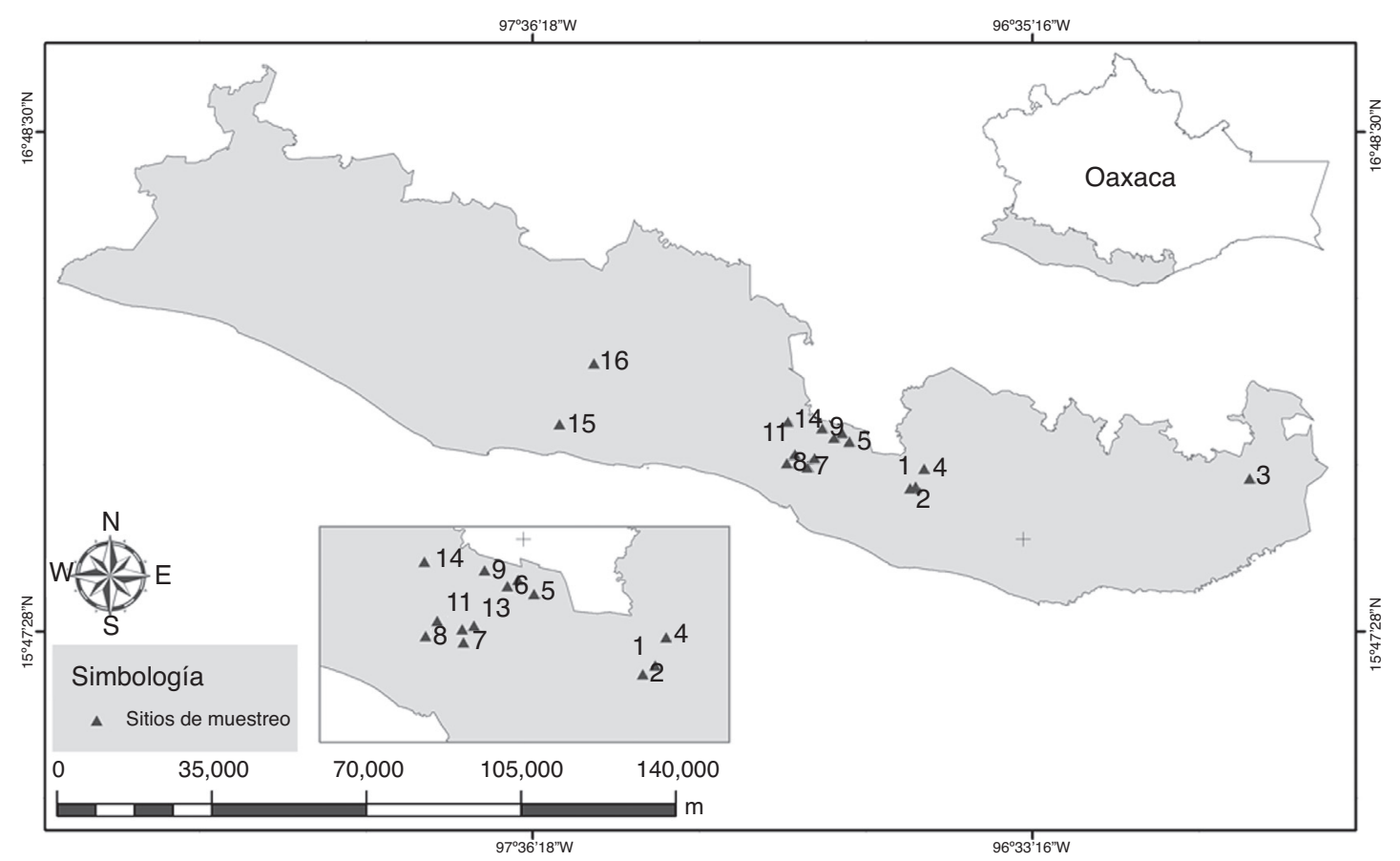

Figura 1. Área de estudio y sitios de muestreo en donde se encontraron poblaciones de Zamia paucijuga y Eumaeus toxea en la provincia fisiográfica Costa de Oaxaca. Las coordenadas y el tipo de vegetación de los sitios están en la tabla 1.

Cuando emergieron, las larvas se alimentaron masticando el envés y el mesófilo, siguiendo las nervaduras hacia la base de la hoja dejando la cutícula del haz; a partir del tercer subestadio larval mastican la hoja completa. Las larvas puparon en el envés de las hojas formando un grupo compacto. Cuando ovipositaron en los estróbilos femeninos, al eclosionar las larvas se introdujeron al estróbilo por la apertura entre las microesporofilas y se alimentaron de la parte interna de la misma; a partir del tercer y cuarto subestadio larval también se alimentaron de la parte externa de la microesporófila y la sarcotesta de la semilla. Para pupar abandonaron el estróbilo y crisalidaron en las hojas. Una cohorte de larvas completó su ciclo biológico consumiendo de 4 a 5 foliolos. Durante el periodo de estudio (junio 2010-mayo 2011), se pudieron observar varias cohortes en una planta, llegando a consumir hasta el $48 \%$ del área foliar de la misma. Únicamente en la población ubicada en selva mediana subcaducifolia conservada, las larvas se alimentaron del raquis de la hoja dejando una galería, generalmente

Tabla 1

Ubicación geográfica, altitud, número de plantas totales, plantas con signos de herbivoría, porcentaje promedio de herbivoría y tipo de vegetación asociada en cada sitio de muestreo de Zamia paucijuga.

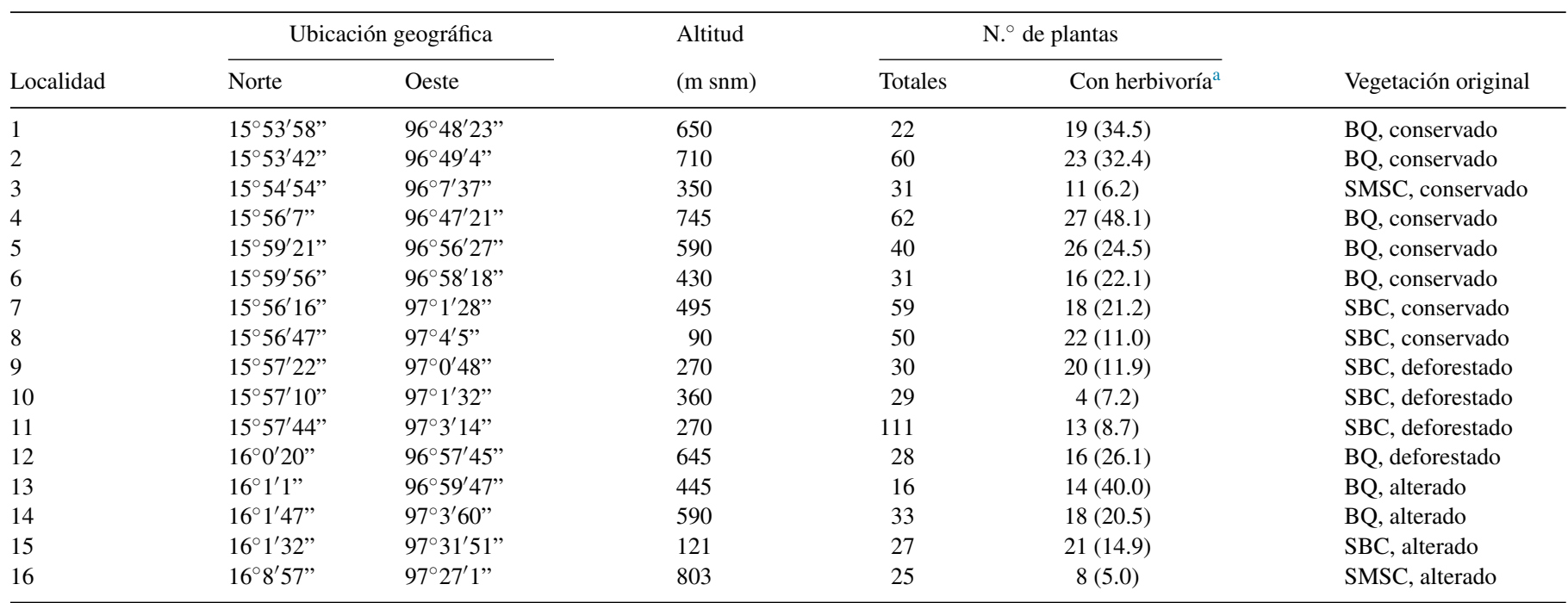

BQ: bosque de encino; SBC: selva baja caducifolia; SMSC: selva mediana subcaducifolia.

${ }^{\text {a }}$ Los números entre paréntesis indican el porcentaje promedio de herbivoría. 


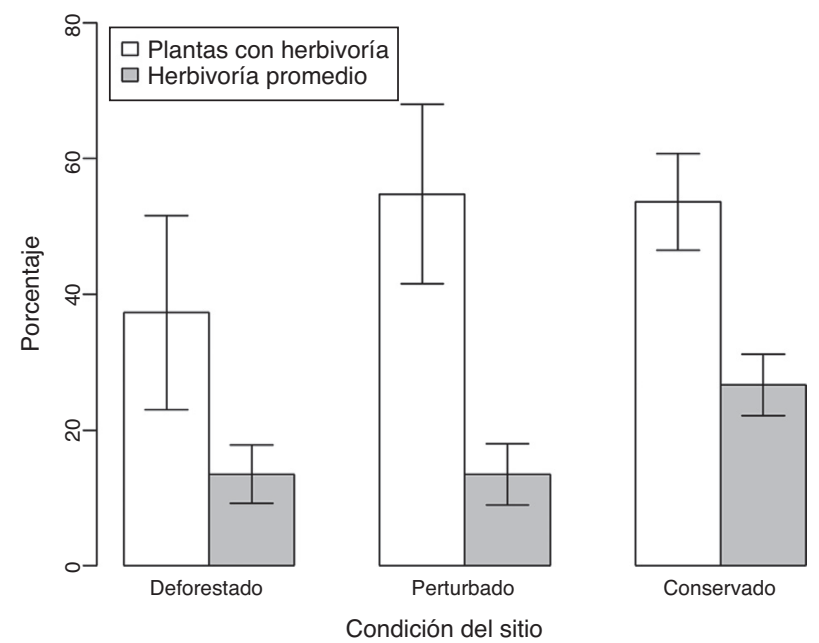

Figura 2. Porcentaje de plantas con signos de herbivoría y porcentaje promedio de herbivoría en cada localidad de Zamia paucijuga.

a partir de la mitad de la hoja continuando hacia la base de la misma.

Todas las oviposiciones en las hojas fueron localizadas en el envés, aproximadamente a la mitad de la misma y en un solo grupo, completamente expandidas de la brotación más reciente y no se observaron oviposiciones en brotes tiernos. Durante la temporada de lluvias ovipositaron en promedio $15.1 \pm 0.7$ hue$\operatorname{vos}(n=12$ oviposiciones), mientras que en la temporada seca ovipositaron en promedio $8.4 \pm 0.6$ huevos ( $n=6$ oviposiciones). Las oviposiciones en estróbilos femeninos fueron en promedio $11.7 \pm 0.3$ huevos ( $n=3$ oviposiciones). No se observaron oviposiciones en estróbilos masculinos, posiblemente porque estos ocurrieron en marzo y abril, época de secas en que la densidad poblacional de E. toxea fue baja. La urgencia de los adultos se registró a los $47 \pm 0.7$ días, la cual ocurrió durante las primeras horas de la mañana (tabla 2). La talla de las mariposas (tabla 2) de ambos sexos fueron iguales $(p=0.157)$.

Los estadios de huevo y larva fueron las etapas más críticas para la supervivencia de los individuos de una cohorte, pues solo $2.7 \%$ de los huevos alcanzaron el estadio adulto (fig. 3). La mortalidad en huevo fue de $28.4 \%$ y la del estadio larval fue de $68.6 \%$; en particular, la mortalidad en el primer y segundo

Tabla 2

Duración del ciclo biológico y tamaño promedio de cada uno de los estadios de Eumaeus toxea alimentado con su hospedante natural Zamia paucijuga en condiciones de campo de la localidad 9 .

\begin{tabular}{llcl}
\hline Estadio & & Duración (días) & Tamaño $(\mathrm{mm})$ \\
\hline Huevo & & $4.8 \pm 0.2$ & $2.5 \pm 0.3$ \\
Larva & & \\
& Subestadio 1 & $4.2 \pm 0.4$ & $3.7 \pm 0.3$ \\
& Subestadio 2 & $5.2 \pm 0.4$ & $6.7 \pm 0.3$ \\
& Subestadio 3 & $4.0 \pm 0.6$ & $9.7 \pm 0.3$ \\
& Subestadio 4 & $5.1 \pm 0.4$ & $10.1 \pm 0.1$ \\
& Prepupa & $5.0 \pm 0.3$ & - \\
Pupa & & $19.0 \pm 0.5$ & - \\
Adulto & & $5.0 \pm 0.6$ & $0^{\top}: 53.52 \pm 0.62, \mathrm{~N}=4$ \\
& & & $: 51.73 \pm 0.80, \mathrm{~N}=5$ \\
\hline
\end{tabular}

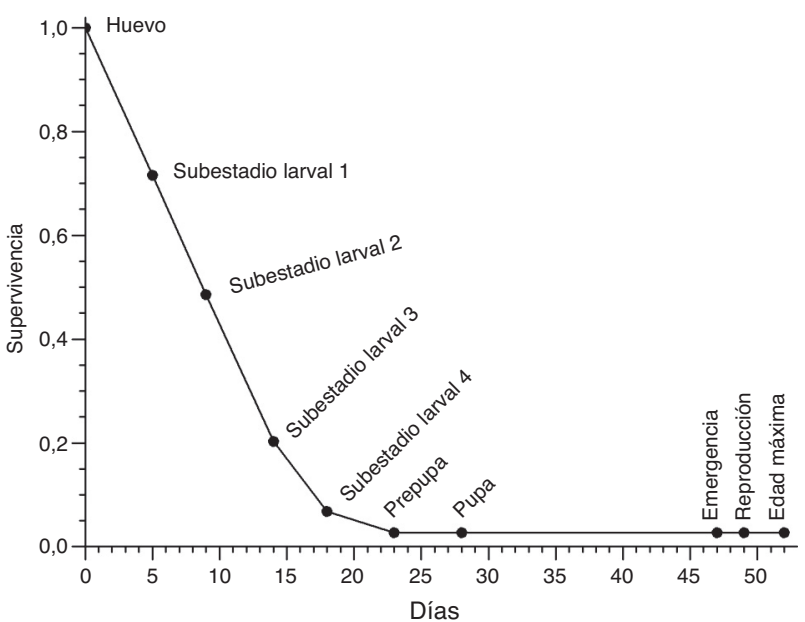

Figura 3. Curva de supervivencia de Eumaeus toxea alimentado con la cícada Zamia paucijuga en la localidad 9, las características de la localidad están en la tabla 1 y su localización en la figura 1 .

subestadio larval fue de 23 y $28.4 \%$, respectivamente. En los huevos se observó falta de eclosión de hasta un $31 \%$ de la cohorte y depredación por un coleóptero del género Dasydactylus (Coleoptea: Erotylidae), cuyos individuos se observaron alimentándose de los exudados durante el proceso de muda de cada uno de los estadios. La tasa neta de reproducción estimada fue de $R_{0}=0.014$ y un tiempo medio en cada generación de $T_{c}=51$ días.

\section{Discusión}

E. toxea es un herbívoro coincidente con la distribución de su hospedante la cícada $Z$. paucijuga, en la provincia fisiográfica Costa de Oaxaca; lo que sugiere que su conservación depende de la preservación de su hospedante y de la cobertura arbórea donde sobreviven las poblaciones. La fluctuación en la densidad poblacional estuvo directamente relacionada con el cambio estacional de una región biogeográfica neotropical; un incremento al inicio de la temporada de lluvias que ocurrió en junio y su disminución al inicio de la temporada de secas que fue en noviembre. Esta fluctuación poblacional fue similar a la observada en especies raras y endémicas como Lycaena hermes Edwards (Lepidoptera: Lycaenidae) (Marschalek y Deutschman, 2008) o de especies especialistas endémicas como Papilio homerus L. (Garraway, Bailey, Freeman, Parnell y Emmel, 2008).

Los individuos de E. toxea que se alimentaron de Z. paucijuga ovipositaron en grupos con más huevos $(15.1 \pm 0.7)$ que aquellas que se alimentaron de Zamia loddigesii Miquel (5.2 \pm 0.5$)$ (Castillo-Guevara y Rico-Gray, 2002), pero menos que Eumaeus godartii Huebn. (= minyas o minijas, Schneider et al., 2002) que ovipositaron 18-57 huevos cuando se alimentan de Z. encephalartoides en Colombia (González, 2004). La talla de las mariposas fue $53.52 \pm 0.62 \mathrm{~mm}$ para machos y $51.73 \pm 0.80 \mathrm{~mm}$ para hembras, ambas tallas fueron semejantes $(p=0.1571$; tabla 2), de manera similar a lo reportado por Martínez-Lendech, Córdoba-Aguilar y Serrano-Meneses (2007) para los ejemplares de la vertiente del golfo, que fue de $25.47 \pm 0.49 \mathrm{~mm}$ para 
hembras y $25.88 \pm 0.35 \mathrm{~mm}$ para machos. Sin embargo, una prueba de comparación por traslape de intervalos del $95 \%$ de confianza indican que las mariposas de este estudio son considerablemente de mayor tamaño que las de la vertiente del golfo (intervalo de confianza para la talla en milímetros del presente trabajo: hembras [50.84, 52.62], machos [51.31, 53.73], y de las de la vertiente del golfo de acuerdo con los datos de MartínezLendech et al. (2007): hembras [24.45, 26.49], machos [25.09, 26.67]).

La mortalidad observada en este estudio fue similar a la reportada cuando se alimentaron de Z. loddigesii; para huevo que fue de $31.25 \%$ ( $p=0.8112)$ y para larva de $64.9 \%$ ( $p=$ 0.6653 ) (Castillo-Guevara y Rico-Gray, 2002). Por la metodología empleada no fue posible observar canibalismo entre las larvas, un factor de mortalidad importante reportado para esta especie (Castillo-Guevara y Rico-Gray, 2002; González, 2004). La mortalidad observada hasta el estadio adulto $(2.7 \%)$ fue similar a la reportada para otras especies de mariposas neotropicales raras, como Hypna clytemnestra huebneri Butler (Nymphalidae: Charaxinae), en la cual el 2.3\% de los huevos alcanzan el estadio adulto (Gomes-Filho, 2003). La tasa neta de reproducción estimada fue considerablemente menor que la tasa de reproducción de Aulascaspis yasumatsui Takagi (Hemiptera: Diaspididae), considerada plaga en las especies del género Cycas (Cycadales: Cycadaceae) en Taiwán, que fue de $R_{0}=111.51$ en un tiempo generacional de 47.24 días (Bailey, Chang, Lai y Hsu, 2010).

No se observaron larvas de E. toxea alimentándose de Dioon holmgrenii De Luca, Sábato y Vázquez-Torres, otra cícada endémica con la cual convive en las localidades 1, 2, 4, 9 y 14. No se observaron estadios larvales diferentes alimentándose de la misma planta como fue el caso de Eumaeus childrenae Gray alimentándose de Ceratozamia mexicana Brongn. y Zamia fischerii en poblaciones de la Huasteca (ContrerasMedina, Ruiz-Jiménez y Luna-Vega, 2003); tampoco se observó alimentándose de plántulas, como fue el caso de Dioon edule Lindl., en Veracruz (Vovides, 1990). Aunque las larvas consumen gran cantidad del tejido foliar durante un año, no ocasionan daño serio a las plantas, pues únicamente ocupan de 4 a 5 foliolos de una hoja para completar su ciclo de vida.

Nuestras observaciones indican que cuando se alimentan de los estróbilos femeninos no ocasionan daño a la semilla, contrario a lo que ocurre cuando se alimentan de estróbilos femeninos de Z. loddigesii (Castillo-Guevara y Rico-Gray, 2002), porque se alimentaron de la sarcotesta durante el proceso de liberación de la misma proporcionándole beneficio. La sarcotesta tiene efecto inhibidor en la germinación (Dehgan, 1983) y su remoción es un procedimiento necesario para su germinación (Calonje, Kay y Griffith, 2011). Aun cuando no se cuentan con estudios de conservación de esta especie de mariposa, es claro que su conservación está directamente relacionada con la preservación de las poblaciones de la cícada hospedante y cobertura arbórea donde sobreviven las poblaciones.

\section{Agradecimientos}

Este trabajo fue financiado parcialmente por el Programa para el Mejoramiento del Profesorado (PROMEP), proyecto
N. ${ }^{\circ}$ 2IG1003 de la convocatoria «Apoyo a la Incorporación de Nuevos PTC 2010».

\section{Referencias}

Bailey, R., Chang, N. T., Lai, P. Y. y Hsu, T. C. (2010). Life table of cycad scale, Aulacaspis yasumatsui (Hemiptera: Diaspididae), reared on Cycas in Taiwan. Journal of Asia-Pacific Entomology, 13, 183-187.

Calonje, M., Kay, J. y Griffith, M. P. (2011). Propagation of cycad collections from seed: applied reproductive biology for conservation. Sibbaldia, 9, 77-96

Carey, J. R. (1993). Applied demography for biologists with special emphasis on insects. New York: Oxford University Press.

Castillo-Guevara, C. y Rico-Gray, V. (2002). Is cycasin Eumaeus minyas (Lepidoptera: Lycaenidae) a predator deterrent? Interciencia, 27, 465-470.

Contreras-Medina, R., Ruiz-Jiménez, C. A. y Luna-Vega, I. (2003). Caterpillars of Eumaeus childrenae (Lepidoptera: Lycaenidae) feeding on two species of cycads (Zamiaceae) in the Huasteca region, Mexico. Revista de Biología Tropical, 51, 201-204.

Dehgan, B. (1983). Propagation and growth of cycads. A conservation strategy. Proceedings of the Florida State Horticultural Society, 96, 137-139.

Garraway, E., Bailey, A. J. A., Freeman, B. E., Parnell, J. R. y Emmel, T. (2008). Population studies and conservation of Jamaica's endangered swallowtail butterfly Papilio (Pterourus) homerus. Journal of Insects Conservation, 12, 383-397.

Gomes-Filho, A. (2003). Seasonal fluctuation and mortality schedule for immatures of Hypna clytemnestra (Butler), an uncommon neotropical butterfly (Nymphalidae: Charaxinae). Journal of Research on the Lepidoptera, 37, $37-45$.

González, F. (2004). Herbivoría en una gimnosperma endémica de Colombia, Zamia encephalartoides (Zamiaceae) por parte de Eumaeus (Lepidoptera: Lycaenidae). Revista de la Academia Colombiana de Ciencias, 28, 233-243.

Inegi, Conabio e INE (Instituto Nacional de Estadística, Geografía e Informática, Comisión Nacional para el Conocimiento y Uso de la Biodiversidad e Instituto Nacional de Ecología. (2008). Ecorregiones terrestres de México. Escala 1:1,000.000. México, D. F.: Inegi.

Marler, T. C. (2010). Cycad mutualist offers more than pollen transport. American Journal of Botany, 97, 841-845.

Marschalek, D. A. y Deutschman, D. H. (2008). Hermes copper (Lycaena [Hermelycaena] hermes: Lycaenidae): life history and population estimation of a rare butterfly. Journal of Insects Conservation, 12, 97-105.

Martínez-Lendech, N., Córdoba-Aguilar, A. y Serrano-Meneses, M. A. (2007). Body size and fat reserves as possible predictors of male territorial status and contest outcome in the butterfly Eumaeus toxea Godart (Lepidoptera: Lycaenidae). Journal of Ethology, 25, 195-199.

Nicolalde-Morejón, F., Vovides, A. P. y Stevenson, D. W. (2009). Taxonomic revision of Zamia in Mega-Mexico. Brittonia, 61, 301-335.

Rivera-Nava, J. L., Velasco-García, M. V., Ruiz-García, N., Ruiz-Jiménez, C. A., Méndez-Pérez, B. Y. y Sánchez-de la Vega, G. (2011). Ciclo y tabla de vida de Eumaeus toxea en Zamia sp. en la costa de Oaxaca. En III Congreso Mexicano de Ecología. 3-7 de abril. Boca del Río, Veracruz, México.

Rodarte-García, R. (1997). Ecosistemas y biodiversidad en la costa oaxaqueña. Acercamiento descriptivo altitudinal. Ciencia y Mar, 1, 44-48.

Salas-Morales, S. H., Saynes-Vásquez, A. y Schibli, L. (2003). Flora de la costa de Oaxaca, México: lista florística de la región Zimatán. Boletín de la Sociedad Botánica de México, 72, 21-58.

Schneider, D., Wink, M., Sporer, F. y Lounibos, P. (2002). Cycads: their evolution, toxins, herbivores and insect pollinators. Naturwissenschaften, 89, 281-294.

Semarnat (Secretaría de Medio Ambiente y Recursos Naturales). (2010). Norma Oficial Mexicana Nom-059-Semarnat-2010, Protección ambiental-Especies nativas de México de flora y fauna silvestres-Categorías de riesgo y especificaciones para su inclusión, exclusión o cambio-Lista de especies en riesgo. Diario Oficial de la Federación. 30 de diciembre de 2010. Segunda Sección. México. 
Stevenson, D. W., Norstog, K. J. y Fawcett, P. K. S. (1998). Pollination biology of cycads. En S. J. Owens y P. J. Rudall (Eds.), Reproductive biology (pp. 277-294). Kew: Royal Botanic Gardens.

Terry, L. I., Walter, G. H., Donaldson, J. S., Snow, E., Forster, P. I. y Machin, P. J. (2005). Pollination of australian macrozamia cycads (Zamiaceae): effectiveness and behavior of specialist vectors in a dependent mutualism. American Journal of Botany, 92, 931-940.

Torres-Colín, R. (2004). Tipos de vegetación. En A. J. García-Mendoza, M. J. Ordoñez y M. Briones-Salas (Eds.), Biodiversidad de Oaxaca (pp. 105-117). México, D. F: Instituto de Biología, UNAM-Fondo Oaxaqueño para la Conservación de la Naturaleza-World Wildlife Fund.

Velasco-García, M. V., Sánchez-De la Vega, G. y Molina-García, E. (2011). Distribución y estado actual Zamia paucijuga en la costa y sierra Sur de Oaxaca. En III Congreso Mexicano de Ecología. 3-7 de abril. Boca del Río, Veracruz, México.

Vovides, A. P. (1990). Spatial distribution, survival, and fecundity of Dioon edule (Zamiaceae) in a tropical deciduous forest in Veracruz, Mexico, with notes on its habitat. American Journal of Botany, 77, 1532-1543. 